\title{
Finite Element Analysis of Shield Pipe Structure and Stress Cone Position of 10kV Cable
}

\section{Intermediate Joint}

\author{
Bo-yu Shang 1,* , Rui-ming Fang ${ }^{2}$ \\ ${ }^{1}$ College of Information Science and Engineering, Huaqiao University, 361021 Xiamen, China \\ ${ }^{2}$ College of Information Science and Engineering, Huaqiao University, 361021 Xiamen, China
}

\begin{abstract}
The shielding tube and stress cone of the intermediate joint of XLPE cable accessory make the potential distribution along the insulation surface linearized to optimize the electric field and. By establishing the finite element model of the intermediate joint of $10 \mathrm{kV}$ cable accessory, the electric field distribution of different structure shielding tube and stress cone in different position of the joint is simulated and analyzed. The results show that the structure of shield tube and the position of stress cone are also a parameter characteristic which can not be ignored in the design, and they have a great influence on the distribution of the field strength of the intermediate joint. On the basis of finite element analysis, the optimum shape of shield tube and the best position of stress cone are obtained by comparing and analyzing the variation law of electric field intensity.
\end{abstract}

\section{Introduction}

Cable accessory plays an important role in the connection and transition of power transmission lines, and is an indispensable part to ensure the normal operation of power system. At present, the research of cable accessories at home and abroad mainly focuses on the effect of artificial defects (knife marks, burr tips, metal particle suspension, etc.) on the electric field distribution of the accessories[1]. However, the influence of different

\footnotetext{
* Corresponding author: fangrm@126.com
} 
distances between different types of shielded tubes and stress cones and shield tubes on cable attachments is rarely reported[2-5].

The stress cone can significantly improve the field strength concentration at the edge of the metal sheath and the shielding layer, so that the field strength tends to be evenly distributed[6], and avoid problems such as temperature rise caused by local electrical stress concentration, aging of insulating materials and even breakdown of cable joints in long-term operation of cable joints. When the shielded tube at the middle joint of the cable is destroyed, the shielding effect of the electric field is weakened to some extent, and the external field strength increases, which has a great influence on the steady operation of the accessories.

\section{Model analysis and finite element method}

\section{1 finit element method}

Finite element method is a numerical method based on variational principle and subdivision difference value, which is similar to the finite difference method for solving boundary value problems[7-8].

For the first boundary $\left.\varphi\right|_{c}=f(S)$, And satisfying the Poisson equation: $\nabla^{2} \varphi=-\rho / \varepsilon$, of which $\varphi$ are potential, $c$ is boundary condition, $f(S)$ is a point function of boundary point $S, \rho$ is space charge density, $\varepsilon$ as dielectric constant, Let the total electric field energy in the space field be $W$, The minimum energy of the electric field is:

$$
\frac{\partial W}{\partial \varphi_{i}}=\frac{\partial}{\partial \varphi_{i}}\left(\frac{1}{2} \Phi^{T} \mathbf{K} \Phi\right)=0, \quad i=1,2, \ldots, \mathrm{n},
$$

Where $\varphi_{i}$ is node potential, $\Phi$ is the column vector of the boundary node and the inner node potential, $\mathbf{K}$ is the matrix of electric field energy coefficient of boundary node and inner node. Because the boundary node potential is known, disregarding formula (1), for $n$ inner node, $n$ element linear equations can be obtained:

$$
\sum_{j=1}^{n_{0}} k_{i j} \varphi_{i}=0, \quad i=1,2, \ldots, \mathrm{n},
$$

Where $k_{i j}$ is $i$ row and $j$ column coefficients of $\mathbf{K}$, move the known term of the boundary node voltage of formula (2) to the right of the equation:

$$
\sum_{j=1}^{n} k_{i j} \varphi_{j}=\sum_{j=n+1}^{n_{0}} k_{i j} \varphi_{j}, \quad i=1,2, \ldots, \mathrm{n},
$$

Express formula (3) in the form of a matrix: $\mathbf{K}_{i n 1} \Phi_{i n 1}=\mathbf{B}$, Where $\Phi_{i n 1}$ is the internal position vector, $\mathbf{K}_{i n 1}$ is the inner node energy coefficient matrix, $\mathbf{B}$ is a constant sequence. The numerical solution of the boundary value problem is obtained. 


\section{2 model analysis}

In this paper, the YJV22-8.7/15-3×185 type single-core cable and its matched connector with a length of $120 \mathrm{~mm}$ are taken as the research object. In this paper, a two-dimensional axisymmetric model is used, according to its shape parameters, the 1:1 scale modeling and simulation based on ANSYS software is carried out, and the simulation analysis is carried out by drawing in APDL language.

In this paper chooses to simulate in electrostatic field environment. At the same time, the two cable cores are connected by connectors, which are regarded as the direct connection of the same materials during the simulation process, and the disconnection is ignored. Figure 1 shows the $1 / 4$ structure model of the $10 \mathrm{kV}$ cold-shrink cable intermediate joint.

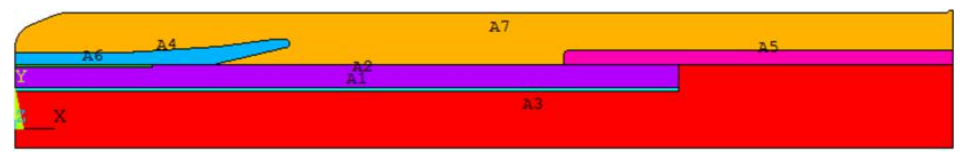

Fig. 1. 1/4 structure model of the $10 \mathrm{kV}$ cold-shrink cable intermediate joint.

A1 is the inner semi-conductive layer, A2 is the main insulation layer, A3 is the cable core, A4 is the stress cone, A5 is the shielded tube, A6 is the outer semi-conductive layer, A7 is the main insulation of accessories. The relative permittivity of intermediate conductor, accessories main insulation, semiconductive layer, shield tube, stress cone, cable main insulation respectively is $10000,2.8,60,700,28,2.3$.

\subsection{Finite element simulation analysis boundary condition}

In order to study the electric field distribution of cable accessories, it is necessary to set the boundary conditions. The effective value of rated voltage of $10 \mathrm{kV}$ crosslinked polyethylene cable intermediate joint is $U_{0}=8.7 \mathrm{kV}$. Considering the voltage difference of 1.1 times between the first end and the end of the transmission line, and The conversion factor between the effective value of $\mathrm{AC}$ voltage and the peak value is $\sqrt{2}$. The voltage applied above the cable core is $13.5 \mathrm{kV}$ [9-10].

At the same time, according to the installation specification, the cable copper tape shield is connected with ground wire when the intermediate joint is installed, so the contact surface between the outer semi-conductive layer and the stress cone is zero potential.

\section{Simulation result analysis}

\subsection{Electric Field Simulation Analysis of different shielding Tube structures}


The simulation calculation of six kinds of shielding tubes with different shapes in the middle joint of cable is carried out.

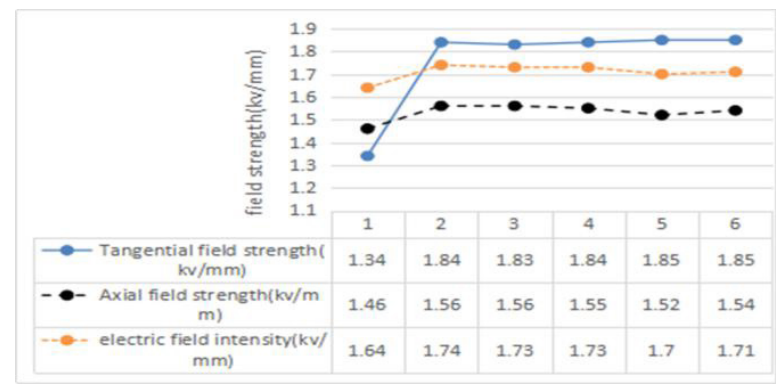

Fig. 2 Variation of Electric Field intensity of shielding Tube with different shapes

In Fig. 3, 1, 2, 3, 4, 5, and 6 respectively represent the shape of the shielding tube is the ends of the shielding tube are 1/4 arc, and the central angle of the end is 90 degrees, 60 degrees, 45 degrees, 30 degrees, and 0 degrees. The electric field simulation results show that the electric field intensity at the shield tube is the smallest when the end is $1 / 4$ circular arc. It can optimize the field strength and is the best choice. Many cable accessory manufacturers often use a very simple treatment, that is, cut the two ends of the middle shield tube into an acute angle, in fact, it can not improve the field strength, but consider the economic treatment. If the service life of cable accessories is more scientifically considered, the shielding pipe structure should also be taken into account in the design of accessories.

\subsection{Simulation analysis of electric field of stress cone at different positions}

The stress cones of six different positions of the cable joint intermediate joint are simulated and calculated.

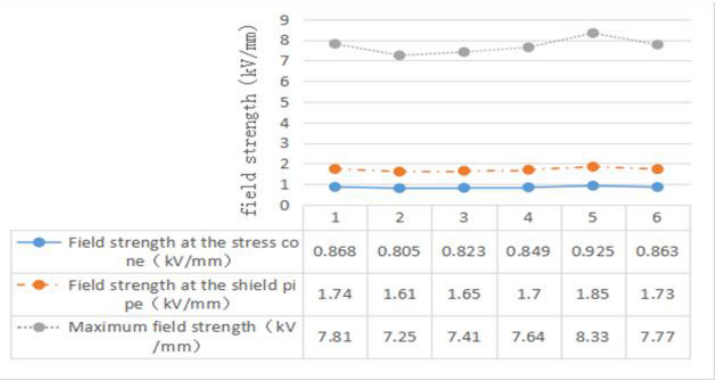

Fig. 3 Variation of electric field intensity at different distances between stress cone and shield tube

In Fig. 3, 1, 2, 3, 4, 5, 6 respectively represent the distance between stress cone and shielding tube as $60 \mathrm{~mm}, 50 \mathrm{~mm}, 40 \mathrm{~mm}, 30 \mathrm{~mm}, 20 \mathrm{~mm}$ and $10 \mathrm{~mm}$. It can be seen from the electric field simulation results that the field strength at the stress cone, the field strength at 
the shielding tube and the maximum field strength are the smallest when the distance between the stress cone and the shielding tube is $50 \mathrm{~mm}$. Therefore, the field strength can be optimized when the distance is $50 \mathrm{~mm}$, which is the best choice. At the same time, the distance between the stress cone and the shield tube will increase when the distance between the stress cone and the shield tube continues to decrease, so the distance should not be shortened in order to save the material in the design of the cable accessory.

\subsection{The combined of shielding tube structure and stress cone position}

The simulation analysis of the interaction between different shielding tube structures and stress cone positions is carried out. The optimal selection of the shielding tube structure and the stress cone position is found through analysis.
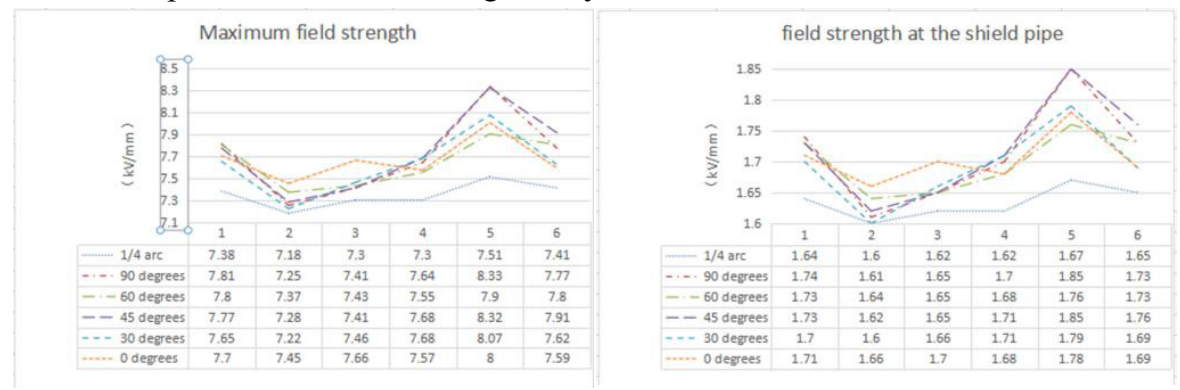

Fig. 4 The variation of field strength for different shielding tube structures and stress cone locations

Through finite element analysis, the simulation results of different shielding tube structures and stress cones are shown in figure 4. In Fig. 4, 1, 2, 3, 4, 5, 6 respectively represent the distance between stress cone and shielding tube as $60 \mathrm{~mm}, 50 \mathrm{~mm}, 40 \mathrm{~mm}$, $30 \mathrm{~mm}, 20 \mathrm{~mm}$ and $10 \mathrm{~mm}$. The first figure is the maximum field strength variation rule for the combination of different shielding tube structures and stress cone positions. the second picture is the variation of field strength at the shielding tube for the combination of different shielding tube structure and stress cone position. According to the simulation results of fig. 4, the maximum field intensity and field strength at the shield pipe is minimum when the distance between the stress cone and the shielded tube is $50 \mathrm{~mm}$, and the end of the shield tube is $1 / 4$ arc.

\section{Conclusion}

When the tip is $1 / 4$ arc, the tangential field strength and the axial field strength at the shield tube are the smallest. Therefore, the $1 / 4$ arc shape at the end of the shielding tube can optimize the field strength, which is the best choice. If the service life of cable accessories is more scientifically considered, the shielding pipe structure should also be taken into account in the design of accessories. 
The field strength at the stress cone, the field strength at the shielding tube and the maximum field strength are the smallest when the distance between the stress cone and the shielding tube is $50 \mathrm{~mm}$. Therefore, the field strength can be optimized when the distance is $50 \mathrm{~mm}$, which is the best choice. At the same time, the distance between the stress cone and the shield tube will increase when the distance between the stress cone and the shield tube continues to decrease, so the distance should not be shortened in order to save the material in the design of the cable accessory.

In cable attachment, the end of shield tube is $1 / 4$ arc, and the distance between stress cone and shield tube is $50 \mathrm{~mm}$, which can optimize the field strength and is the best choice.

\section{References}

1. H. Orton, HVT. 41, 1057-1067(2015)

2. G. Song, A. Atrens, HVT. 39, 1981-2004( 2003)

3. B. J. Harrison, IEEE. 7, 1-6(2002)

4. H. Xiang, STI. 30, 7(2015)

5. W. Pan, Z. W. Chen, Q. U. Shao, Guangxi EP, 2(2012)

6. W. Chao, Y. G. Liu, L. Gang, HVE. 33, 152-154(2007)

7. I. Lindell, OUP, 16(1992)

8. X. Zhang, Y. Yang, Sichuan EPT, 4(2014)

9. G. J. Liu, J. Xiong, B. Y. Ren, Guangdong EP, 2(2011)

10. G. Liu, B. Y. Ren, Chen Z, IM, 3(2011) 